\title{
Brote de enfermedad diarreica aguda causado por Shigella flexneri en una escuela de Madrid, Cundinamarca: caracterización fenotípica y genotípica de los aislamientos
}

\author{
Marylin Hidalgo ${ }^{1}$, María Elena Realpe ${ }^{1}$, Nélida Muñoz ${ }^{1}$, Diego Sicard ${ }^{2}$, Esperanza Silva ${ }^{3}$, \\ Clara Inés Agudelo ${ }^{1}$, Elizabeth Castañeda ${ }^{1}$ \\ ${ }^{1}$ Grupo de Microbiología, Instituto Nacional de Salud, Bogotá, D.C., Colombia. \\ ${ }^{2}$ Instituto del Seguro Social, Madrid, Cundinamarca, Colombia. \\ ${ }^{3}$ Laboratorio de Salud Pública de Cundinamarca, Bogotá, D.C., Colombia.
}

La shigelosis es una enfermedad diarreica aguda (EDA) que causa alta morbimortalidad en países en vías de desarrollo. En 1997, el Grupo de Microbiología inició un programa en red con los Laboratorios de Salud Pública (LSP) del país para la vigilancia de los principales patógenos causantes de la EDA. Como actividad de este programa, en mayo de 2001, el LSP de Cundinamarca estudió e informó un brote de intoxicación alimentaria en una comunidad escolar en Madrid. El objetivo de este estudio fue caracterizar con técnicas fenotípicas y genotípicas los aislamientos recuperados en el brote, con el fin de establecer la relación clonal entre ellos. Se realizaron coprocultivos en 22 de 195 individuos afectados; los aislamientos se identificaron bioquímica y serológicamente y se determinó el patrón de susceptibilidad antimicrobiana a cloranfenicol, trimetoprim-sulfametoxasol (SXT), tetraciclina, cefotaxima, gentamicina, ampicilina y ciprofloxacina. Se realizó electroforesis en gel de campo pulsado (PFGE) según la metodología descrita por los Centers for Disease Control and Prevention (CDC) de Atlanta, con el empleo de la enzima de restricción Xbal y se utilizó como cepa control Shigella sonnei CDC F2353 y como marcador de peso molecular el fago lambda. En $15(68,2 \%)$ pacientes se identificó Shigella flexneri serotipo 6, biotipo Newcastle, con patrón de resistencia a cloranfenicol, SXT y tetraciclina. La PFGE reveló que $3(20 \%)$ aislamientos fueron idénticos (distancia genética de $100 \%$ ) y los $12(80 \%)$ restantes estuvieron estrechamente relacionados (distancia genética de 86 a 100\%). El sistema de vigilancia en red con los LSP permitió recuperar los aislamientos y los estudios fenotípicos y genotípicos permitieron establecer la relación clonal de los aislamientos involucrados en el brote.

Palabras clave: intoxicación alimentaria, shigelosis, brote de EDA, PFGE, relación clonal.

Outbreak of acute diarrhoeal disease caused by Shigella flexneri: phenotypic and genotypic characteristics of the isolates

Shigellosis is an acute diarrhoeal disease that is the main cause of morbidity and mortality in developing countries. In 1997, the Colombian Instituto Nacional de Salud Microbiology Group organized a network surveillance program with the country's Public Health Laboratories (PHLs) to monitor the principal etiological agents responsible for acute diarrhoeal disease. In May, 2001, the PHL of the state of Cundinamarca reported a food poisoning outbreak involving an elementary school community. The main goal of the Microbiology Group involvement was to establish the molecular relationships among the isolates from the outbreak by phenotypic and genotypic methods of characterization. Stool cultures were obtained from 22 of 195 affected individuals. The Microbiology Group confirmed the identification of the isolates by biochemical and serological probes. The antimicrobial susceptibilities were tested against the following battery of antibiotics: chloramphenicol, trimehoprim-sulfamethozazole, cefotaxime, gentamicin, ampicilin and ciprofloxacin. The isolates were subjected to pulsed field gel electrophoresis (PFGE) using the following CDC (U.S. Centers for Disease Control) protocols: Xbal restriction enzyme, Shigella sonnei CDC F2353 as the reference standard, and lambda phage as a molecular weight marker. 
In 15 of $22(68 \%)$ stool cultures Shigella was recovered, all isolates were identified as Shigella flexneri serotype 6 biotype Newcastle with the same antimicrobial susceptibility profile. PFGE showed that $3(20 \%)$ isolates were identical (100\% genetic similarity) and the other $12(80 \%)$ were very closely related (genetic similarity between $86-98 \%$ ). The network system permitted the INS ready access to the isolates and the implementation of the PFGE permitted a quantitative characterization of the clonal relationship among the isolates from the outbreak.

Key words: Shigella, PFGE, outbreak, molecular epidemiology, food poisoning.

La shigelosis es una enfermedad diarreica aguda, de tipo inflamatorio, ocasionada por Shigella sp.; es considerada como una de las principales causas de morbilidad y mortalidad en niños con diarrea en países en vía en desarrollo $(1,2)$. Según la Organización Mundial de la Salud, el número anual de episodios de infección por Shigella sp. que se producen en todo el mundo se estima en 164,7 millones de casos, 163,2 $(99,1 \%)$ en países en desarrollo y $1,5(0,9 \%)$ en los países desarrollados (1). La mortalidad total asociada con Shigella en los países en desarrollo se estima en 1,3 millones. De estos episodios, el $69 \%$ ocurren en niños menores de cinco años y constituyen el $61 \%$ de todas las defunciones en ese grupo de edad (1).

La shigelosis se caracteriza por la presencia de diarrea acuosa sanguinolenta, acompañada de fiebre y calambres abdominales y puede progresar a una disentería clásica con heces que contienen sangre, moco y pus (2). Los alimentos - el agua contaminadas con la bacteria constituyen el principal factor de riesgo para adquirir la infección; adicionalmente, el hacinamiento y las pobres condiciones de higiene permiten la transmisión de persona a persona y, por tanto, la mayor posibilidad de diseminación $(2,3)$.

Con base en los antígenos somáticos en el género Shigella, se han descrito 49 serotipos y cuatro serogrupos: el serogrupo $A(S$. dysenteriae), el B (S. flexneri), el C (S. boydii) y el D (S. sonnei) (4). Los cuatro serogrupos de Shigella sp. pueden causar enfermedad, ya sea como casos esporádicos o en brotes $(2,3,5)$.

Correspondencia:

Marylin Hidalgo, Grupo de Microbiología, Instituto Nacional de Salud, Avenida Calle 26 \#51-60, Bogotá, D.C., Colombia; teléfono 220 7700, extensión 445; hidalgomary@yahoo.com

Recibido: 21/05/02; aceptado: 04/09/02
Son pocos los datos publicados sobre la prevalencia de Shigella como agente causal de EDA en Colombia; en un estudio realizado en el Valle del Cauca por Newell y colaboradores en 1976, se informó una positividad del 1,3\% en 837 coprocultivos realizados; de las 11 Shigella spp. aisladas, $45,6 \%$ fueron $S$. boydii; $27,2 \%, S$. flexneri y $27,2 \%$ otros serogrupos (6). En otro estudio realizado en Antioquia, de 1993 a 1996, se encontró una positividad de $2,7 \%$ en 874 muestras de pacientes con EDA (7); sin embargo, no se determinó el serogrupo. Recientemente, los datos obtenidos de 1997 a 2001 en el programa de vigilancia por el laboratorio de los serogrupos y susceptibilidad antimicrobiana de los patógenos causantes de EDA que realiza el Grupo de Microbiología con los Laboratorios de Salud Pública del país (LSP), señalan que de 560 aislamientos de Shigella sp. 55\% son S. flexneri; $40 \%$, S. sonnei; 0,8\%, S. dysenteriae; $3,2 \%$, S. boydii, y $1 \%$ no serotipificables. El $60,3 \%$ de estos aislamientos se han recuperado de niños menores de 5 años $(8,9)$.

Para la vigilancia de estos patógenos y, especialmente, para el estudio de los brotes epidémicos es necesario emplear una serie de marcadores fenotípicos como lo son el biotipo, el serotipo y el antibiotipo, entre otros (10). No obstante, estas técnicas adolecen de limitaciones por lo que es necesario interpretar con prudencia sus resultados. Recientemente, la aplicación de las técnicas de biología molecular, con las cuales se estudia directamente el ADN, ha permitido complementar el conocimiento de la epidemiología de las enfermedades infecciosas $(10,11)$.

En este artículo se describe un brote por Shigella flexnerique se presentó en mayo de 2001 en una comunidad escolar, el cual afectó una parte considerable de la población expuesta. El objetivo 
de este trabajo fue caracterizar por técnicas fenotípicas y genotípicas los aislamientos recuperados de los pacientes que sufrieron la intoxicación, con el fin de establecer si existía una relación clonal entre ellos.

\section{Materiales y métodos}

Lugar: el 24 de mayo de 2001 se presentó una intoxicación alimentaria en una concentración escolar del municipio de Madrid, Cundinamarca, 12 horas después de que el personal recibió alimentos en el restaurante de la concentración.

La concentración tiene dos jornadas académicas, una en la mañana a la cual asisten 350 alumnos y una en la tarde con 230 alumnos; además, cuenta con 22 docentes, 1 ecónoma y 2 auxiliares de cocina para un total de 605 personas.

Estudios clínicos, microbiológicos y epidemiológicos: de acuerdo con el informe suministrado por la Oficina de Epidemiología de la Secretaría de Salud de Cundinamarca, las personas afectadas fueron atendidas y tratadas en el Hospital Santa Matilde, en el centro de Atención Ambulatorio del Seguro Social y en el Centro de Salud de El Sosiego de la Secretaría de Salud de Madrid, donde se les realizó una evaluación clínica.

Se llevó a cabo una visita al restaurante escolar y a los establecimientos donde se adquirieron los alimentos. Se tomó una muestra de agua de uno de los grifos de la concentración y 4 salchichas de un paquete de 2,5 libras suministrado por el proveedor, las cuales se enviaron al LSP de Cundinamarca para hacer el estudio de coliformes y enteropatógenos.

Por otra parte, se indagó acerca de la dieta y se obtuvieron datos demográficos que incluyeron edad y género; se evaluaron los procedimientos de limpieza del restaurante de la concentración escolar. Además, se realizaron visitas a los establecimientos en donde fueron adquiridos los alimentos.

En 195 pacientes se realizó un examen coprológico; de 22 (11,3\%) se obtuvo una muestra de materia fecal para coprocultivo. Las muestras fueron procesadas por el LSP de
Cundinamarca donde se realizaron los procedimientos de identificación inicial.

Los 15 aislamientos obtenidos de los coprocultivos, identificados como Shigella sp. fueron enviados al Grupo de Microbiología del INS para confirmación, serotipificación y determinación de la susceptibilidad antimicrobiana.

La confirmación se realizó con las técnicas bioquímicas estandarizadas; la serotipificación se hizo por aglutinación en lámina con antisueros de grupo y de serotipo (Difco) $(4,5,13)$; la susceptibilidad antimicrobiana se determinó por el método de Kirby-Bauer a ampicilina (10 $\mu \mathrm{g})$, cloranfenicol $(30 \mu \mathrm{g})$, trimetoprim-sulfametoxasol SXT $(1,25 / 23,75 \mu \mathrm{g})$, tetraciclina $(30 \mu \mathrm{g})$, ciprofloxacina $(5 \mu \mathrm{g})$, gentamicina $(10 \mu \mathrm{g})$, cefotaxima $(30 \mu \mathrm{g})$ y ácido nalidíxico $(30 \mu \mathrm{g})$, (Becton Dickinson, USA); se utilizaron los parámetros de lectura e interpretación de la NCCLS (14).

PFGE: se realizó con el procedimiento estandarizado en el CDC en el programa de vigilancia de las enfermedades ocasionadas por ingestión de alimentos contaminados, el cual es conocida como PulseNet, The National Molecular Subtyping Network for Foodborne Disease Surveillance $(15,16)$. Las colonias se obtuvieron de un cultivo de 18 horas en agar Luria Bertani (LB) y se suspendieron en solución tamponada (Tris $100 \mathrm{mM}$, EDTA $100 \mathrm{mM}$, pH 8,0) hasta obtener una lectura de 1,3-1,4 DO a una longitud de onda de $610 \mathrm{~nm}$. Para la elaboración de los discos, la suspensión celular se mezcló con agarosa de bajo punto de fusión (Bio-Rad) y 0,5 $\mathrm{mg} / \mathrm{ml}$ de proteinasa $\mathrm{K}$ (Boehringer Manheim). Posterior-mente, los discos se sometieron a lisis celular en una solución tamponada (Tris $50 \mathrm{mM}$, EDTA $50 \mathrm{mM}$, pH 8,0, sarcosil 1\%, proteinasa $\mathrm{K}$ a $0,1 \mathrm{mg} / \mathrm{ml}$ ) a $54^{\circ} \mathrm{C}$ en un baño de María con agitación constante a 200 rpm por un período de 2 horas, después se realizaron 5 lavados con TE (Tris $10 \mathrm{mM}$, EDTA $1 \mathrm{mM}$ ) a $50^{\circ} \mathrm{C}$ por 15 minutos cada lavado. Para la digestión in situ del ADN cromosómico de Shigella embebido en la agarosa se utilizó la enzima de restricción Xbal (Promega). Para la realización del gel se utilizó agarosa 1\% $\mathrm{p} / \mathrm{v}$ (PFGE-grade, Bio-Rad). La electroforesis se 
realizó en el equipo CHEF DR II (BioRad) con un tiempo inicial de $2,2 \mathrm{~s}$, tiempo final $54,2 \mathrm{~s}$, voltaje $6 \mathrm{~V} / \mathrm{cm}$, tiempo de corrido $22 \mathrm{~h}$ a $14^{\circ} \mathrm{C}$. Como marcador de peso molecular se empleó el fago lambda (Biolabs, New England).

Después de la electroforesis el gel se tiñó con bromuro de etidio $(1 \mu \mathrm{g} / \mathrm{ml})$ por 30 minutos. El ADN se visualizó en un transiluminador con luz UV y se tomó la foto (Polaroid).

Los patrones de ADN generados por la PFGE se analizaron por dos criterios que incluyeron la visualización del número y la posición de las bandas y la interpretación según el criterio de Tenover et al. (17). Este permite agrupar los aislamientos en indistinguibles, cuando el número y la ubicación de las bandas en la electroforesis es igual, lo que indica que no existen diferencias genéticas entre ellos; estrechamente relacionados, cuando se presentan 2-3 bandas diferentes lo que señala 1 diferencia genética; posiblemente relacionados, cuando hay diferencias en 4-6 bandas lo que señala la existencia de dos diferencias genéticas y aislamientos diferentes cuando existen 7 o más bandas de diferencia entre los aislamientos lo que indica la existencia de 3 o más eventos genéticos diferentes (17). El segundo criterio se realizó con el programa diversidad en las bases de datos (Diversity database, Bio-Rad-Laboratories). La correlación entre las bandas se llevó a cabo teniendo en cuenta únicamente su posición (coeficiente de Dice) y el árbol filogenético fue obtenido por Unweighted Pair Group Method (UPGMA) (18). En el dendograma se consideró, que los aislamientos que presentaban una similitud de $100 \%$ eran idénticos; de $85 \%$ estrechamente relacionados; entre 75-84\% probablemente relacionados, $\mathrm{y} \leq 75 \%$, diferentes.

Se incluyeron para la PFGE, 5 aislamientos de S. flexneri recuperados de coprocultivos (INS Shf321, Shf-323, Shf-248, Shf-260 y Shf-275), con el mismo patrón de serotipificación y susceptibilidad antimicrobiana, con el fin de observar si existía relación genética entre ellos y con los aislamientos del brote. Cuatro de estos aislamientos fueron recuperados en Bogotá, dos de ellos (Shf-321, Shf-323) en el 2001, los dos restantes (Shf-260 y Shf-275) en el 2000; el aislamiento Shf-248 se recuperó en Medellín en 2000. Tres de los aislamientos fueron recuperados de niños menores de 3 años de edad, el cuarto de un paciente de 20 años y del aislamiento restante no se tenía dato de edad. Se empleó como cepa de referencia $S$. sonnei F2353 CDC, gentilmente enviada por E. Ribot del CDC.

\section{Resultados}

De las 605 personas que ingirieron alimentos en el restaurante escolar, se enfermaron 195, lo que representó una tasa de ataque de $32,2 \%$. Todos los pacientes presentaron un cuadro clínico con síntomas comunes como vómito, diarrea, fiebre no cuantificada, escasa tolerancia a la vía oral y dolor abdominal de 12 horas de evolución.

Una vez establecido el diagnóstico clínico, todos los pacientes fueron tratados con sales de rehidratación, trimetoprim-sulfa y acetaminofén. Se procedió al manejo de la comunidad, con educación a los padres de familia para los cuidados en caso de deshidratación y se estableció una vigilancia epidemiológica continua.

Ninguno de los 195 niños afectados requirió hospitalización, los casos agudos fueron manejados en las salas de observación de cada uno de los centros de atención y no se registró ninguna complicación en ellos.

Los 15 aislamientos se confirmaron bioquímicamente como Shigella sp. y al realizar la serotipificación y la biotipificación, se estableció que todos eran $S$. flexneri serotipo 6 , biotipo Newcastle; todos mostraron el mismo patrón de resistencia a cloranfenicol, SXT y tetraciclina y sensibilidad a ciprofloxacina, cefotaxima, ácido nalidíxico, gentamicina y ampicilina. Los 15 aislamientos fueron codificados por el Grupo de Microbiología como INS-Shf 324 a INS-Shf 337 e INS-Shf 340.

La muestra de agua fue negativa para coliformes totales y enteropatógenos y en las salchichas se recuperó Escherichia coli.

En la investigación epidemiológica se encontró que la dieta suministrada el día antes de la intoxicación fue torta de habichuelas con pollo, salchicha y zanahoria, papa, arroz blanco y agua de panela. 
Se obtuvieron datos demográficos, edad y género, únicamente de los 15 pacientes a quienes se les aisló Shigella sp. De estos 15 pacientes, 6 $(40 \%)$ tenían entre 5-8 años de edad y $9(60 \%)$ entre 9-12 años; 12 (80\%) pacientes eran del género masculino y $3(20 \%)$ del femenino.

En el restaurante se encontró que la limpieza de los utensilios se realizaba con agua filtrada y el aseo con hipoclorito de sodio (Clorox ${ }^{\circledR}$ ) y con un detergente a base de carbonato y silicato (Axión $\circledast$ ). Los tanques de almacenamiento del agua están al alcance de los niños y no se encuentran tapados. Hay dos vendedores de comestibles, uno que atiende la jornada de la mañana y el otro la de la tarde, pero no se pudieron entrevistar.

En la visita realizada a uno de los establecimientos donde se obtuvieron los alimentos (expendio de salchichas) no se logró identificar el lote del producto ni su marca y, en el otro (el expendio del pollo), de acuerdo con la información suministrada por la ecónoma de la concentración, por la alarma ocasionada en el barrio donde estaba ubicada la concentración escolar, realizaron limpieza del refrigerador y colocaron un producto fresco, de un lote diferente al que había estado implicado en el brote.

La digestión enzimática del ADN de los 15 aislamientos del brote y de los 5 no relacionados generó 17 bandas por PFGE que oscilaron en sus pesos moleculares entre 48,5 y $727,5 \mathrm{~kb}$; de acuerdo con los criterios de Tenover, los 15 aislamientos del brote fueron indistinguibles (figura 1). De igual manera, 2 de los aislamientos de Bogotá (Shf-260 y Shf-275) y 1 de Medellín (Shf-248), recuperados en el 2000, fueron indistinguibles de los aislamientos del brote. Los 2 aislamientos

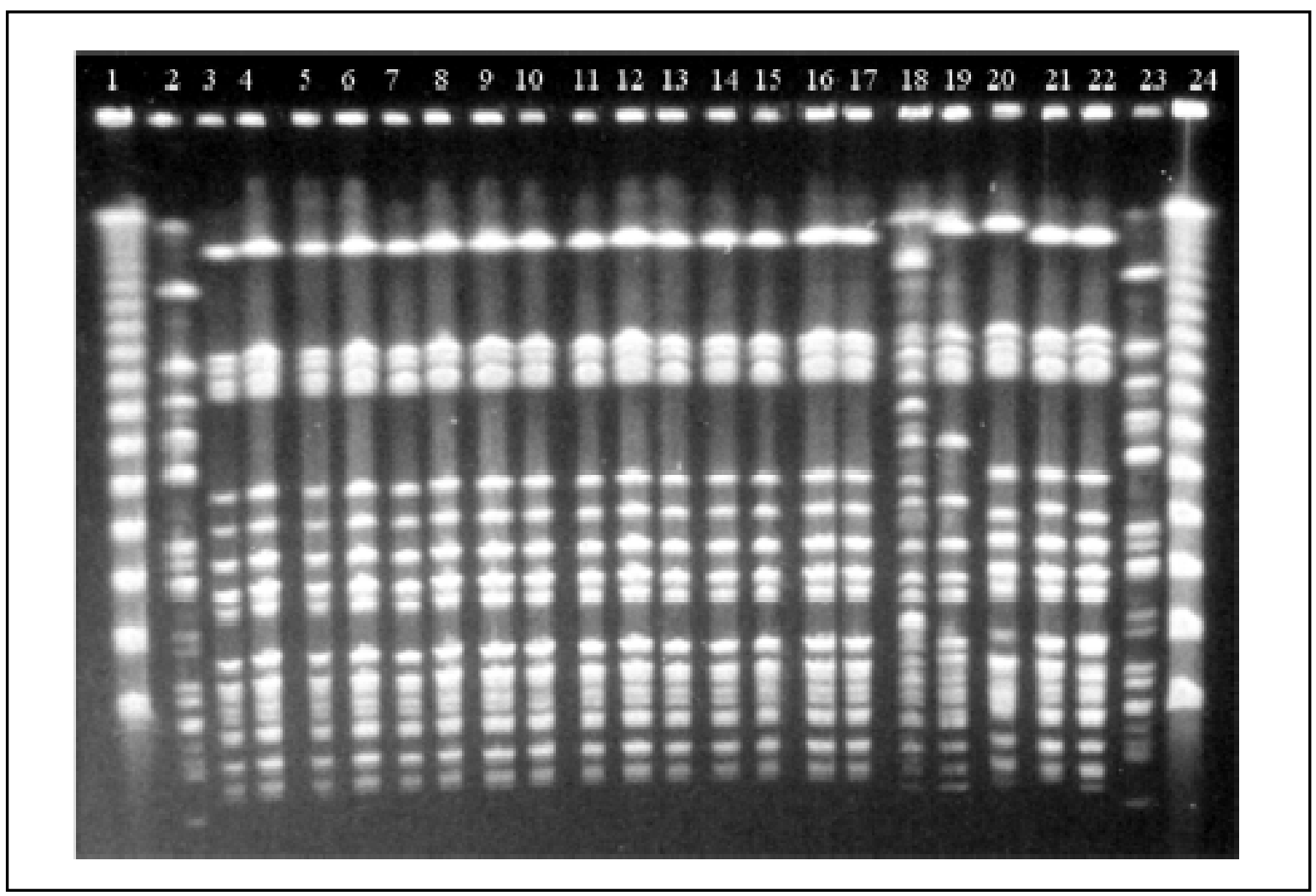

Figura 1. PFGE de Shigella flexneri digerido con la enzima Xbal. Carriles 1 y 24: marcador de peso molecular, fago lambda; carriles 2 y 23: cepa de referencia CDC SF2353; carriles 3-17: aislamientos INS Sh-340, INS Shf-324-INS Shf 337 (aislamientos relacionados con el brote); carriles 18-23 INS Shf-321, INS Shf-323, INS Shf-248, INS Shf-260 y INS Shf275 (aislamientos no relacionados). 
restantes, recuperados en el 2001 se consideraron como diferentes ya que poseían diferencias en más de 7 bandas.

El análisis del dendograma y de la similitud genética reveló que $3(20 \%)$ aislamientos del brote fueron idénticos (similitud genética, 100\%) y $12(80 \%)$ estuvieron estrechamente relacionados (similitud genética entre $86-97 \%$ ). Tres de los aislamientos (Shf-248, Shf-260, Shf275) no relacionados con el brote también presentaron una estrecha relación (similitud genética, 86-97\%). Los dos aislamientos restantes (Shf-321 y Shf-323) no estaban relacionados ya que el análisis reveló una similitud menor del $75 \%$ (figura 2).

\section{Discusión}

Los sistemas de vigilancia en red permiten conocer los serotipos y patrones de susceptibilidad más frecuentes de los enteropatógenos y la introducción de técnicas de biología molecular complementa esta información al establecer la relación clonal de los aislamientos involucrados en brotes (13).

Los aislamientos analizados en este trabajo fueron identificados en el programa de vigilancia, lo cual demuestra la utilidad de estos sistemas

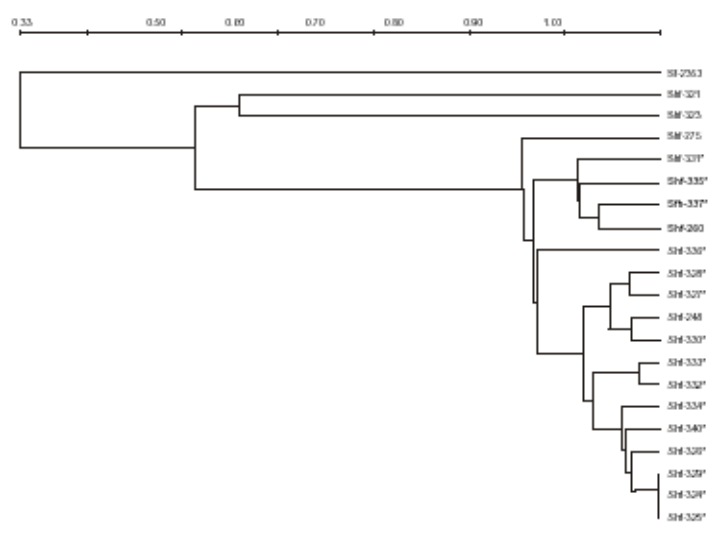

Figura 2. Dendograma obtenido del análisis de la PFGE de 20 aislamientos de Shigella flexneri; 15 aislamientos (INS Shf-340, INS Shf-324 a INS Shf-337), marcados con un asterisco* ${ }^{*}$, fueron recuperados del brote; 5 aislamientos (INS Shf323, INS Shf-323, INS Shf 248, INS Shf-248, INS Shf260 e INS Shf-275) no estuvieron relacionados con el brote y la cepa control S. sonnei (CDC SF2353). Basado en el coeficiente de Dice y construido por UPGMA (DiversityDatabase Bio-Rad). en red, ya que permiten detectar rápidamente los agentes relacionados con un brote. Adicionalmente, el programa de vigilancia en red ha permitido establecer que $S$. flexneri es el principal serogrupo del género Shigella aislado en nuestro país (8).

En Colombia, en 1997, el 50\% de los egresos hospitalarios por EDA eran debidos a Salmonella spp. y Shigella sp. lo cual concuerda con estudios realizados en Brasil en los que se informa como el segundo agente etiológico causante de enfermedad diarreica aguda en niños mayores de dos años, en un grupo de aislamientos obtenidos de 1994 a $1997(19,20)$.

Las intoxicaciones alimentarias, como la descrita en este brote, ocasionadas por Shigella sp. son más comunes en la población que asiste a guarderías, comunidades militares o escuelas, debido a la facilidad de transmisión por los alimentos y el agua, ya que una baja dosis de microorganismos (de 10 a 200 unidades formadoras de colonias) es capaz de producir la infección, aun más si las condiciones de higiene son deficientes y el control de los alimentos no es el óptimo; además, en niños, las deficiencias en los mecanismos de inmunidad intestinal contribuyen a que sea una de las poblaciones más afectadas (2,5,21-24).

En la literatura médica están descritos muchos brotes ocasionados por S. sonnei, S. flexneri, $S$. dysenteriae 1 y en la mayoría de ellos está implicada la población infantil; en países industrializados se ha observado que el serogrupo más frecuente es $S$. sonnei; en contraste, $S$. flexneri es el más frecuente en países en vías de desarrollo, lo que indica que esta patología es de distribución mundial, pero causada por diferentes serogrupos. Todos los aislamientos de este brote correspondieron a $S$. flexneri, lo que concuerda con la literatura $(2,4,5,8,9,23,25)$.

El objetivo de tipificar las bacterias implicadas en un brote es proporcionar al laboratorio y a los epidemiólogos la información sobre la relación genética de los aislamientos $(26,27)$. La PFGE es considerada como la técnica de oro de los métodos de tipificación molecular por su alto poder discriminatorio, buena reproducibilidad intra 
e interlaboratorios, fácil interpretación y costo moderado (13). Con la adición de programas computarizados y escáner de las imágenes es posible crear bancos de datos de patrones de PFGE para todos los microorganismos (13). La PFGE se puede aplicar a pequeños grupos de aislamientos, preferiblemente menor o igual a 30, epidemio-lógicamente relacionados (26). También ha sido empleada para caracterizar geno-típicamente otras especies de Shigella como $S$. sonnei. En este estudio se confirmó el poder discriminatorio de la técnica (26).

Algunos autores como Yuk-Fong Liu et al. (27), señalan como inconvenientes de la técnica el tiempo requerido para su ejecución y la dificultad en su realización (27). Cabe destacar que la técnica estandarizada por la red denominada PulseNet liderada por el CDC y empleada en este estudio no fue considerada como laboriosa ni dispendiosa y se obtuvieron resultados en un tiempo máximo de 24 horas. El objetivo de la red PulseNet es el de incrementar la vigilancia de brotes ocasionados por alimentos contaminados, identificar la fuente de la infección y establecer electrónicamente la comparación de huellas genómicas (15). Otros estudios han confirmado la utilidad de la PFGE no solamente con grupos pequeños de aislamientos, sino en estudios de vigilancia que involucran aislamientos de muchos años, obteniendo excelentes resultados (10, $27,28)$.

Aunque la investigación clínica, epidemiológica y de laboratorio clínico permitió estudiar este brote de EDA y determinar la clonalidad de su agente etiológico, no se pudo identificar la fuente común, lo que no permitió caracterizarlo completamente.

Esto señala la necesidad de mejorar la calidad del sistema de vigilancia de las enfermedades transmitidas por alimentos, para que las diferentes áreas de los LSP trabajen en forma coordinada con la Oficina de Epidemiología de los Servicios de Salud Pública. Así se podrá identificar la causa de las intoxicaciones y desarrollar planes de acción claros y consistentes para el manejo y control de este tipo de brotes y, además, proponer medidas adecuadas para evitar futuros problemas de esta índole.
En resumen, la técnica de PFGE estandarizada en el Grupo permitió establecer una relación epidemiológica y genética entre los aislamientos involucrados en un brote ocasionado por $S$. flexneri en una concentración escolar. También se logró establecer la clonalidad de los aislamientos de $S$. flexneri no vinculados epidemiológicamente con el brote, lo que abre nuevas posibilidades de estudio para generar conocimiento sobre la circulación de clones de S. flexneri en nuestro medio.

\section{Agradecimientos}

Agradecemos especialmente a Efraín Ribot, jefe del laboratorio de desarrollo y validación de metodos de la red de electroforesis de campo pulsado PulseNet, Sección de Enfermedades de Intoxicación Alimentaria y Diarrea de los Centers for Disease Conrol and Prevention (CDC), Atlanta, por el envío de los protocolos de la técnica de PGFE, la cepa control y por sus valiosos comentarios.

\section{Referencias}

1. Kotloff KL, Winickoff JP, Ivanoff B, Clemens JD, Swerdlow DL, Sansonetti PJ et al. Global burden of Shigella infections: implications for vaccine development and implementation of control strategies. Bull WHO 1999;77:651-66.

2. Benenson AS, editor. Shigellosis. En: Control of communicable diseases in man. Fifteenth edition. Washington, D.C.: American Public Health Association; 1990. p.391-4.

3. Posada B. Shigelosis. En: Restrepo A, Robledo J, Bedoya VI, Restrepo M, Botero D et al., editores. Fundamentos de medicina. Quinta edición. Medellín: CIB; 1996. p.430-2.

4. Cherly AB, Frances WB, Joy GW, Nancy AS. Escherichia, Shigella, and Salmonella. En: Murray PR, Baron EJ, Pfaller MA, Tenover FC, Yolken RH, editors. Manual of clinical microbiology. 7th. edition. Washington, D.C.: ASM Press; 1999. p.459-66.

5. Koneman EW, Allen SD, Janda WM, Schreckenberger PC, Winn WC Jr, editors. The Enterobacteriaceae. En: Color atlas and textbook of diagnostic microbiology. 5th Edition. Philadelphia, PA: Lippincott Williams \& Wilkins; 1997. p.171-241.

6. Newell KW, Dover AS, Clemmer DI, D'Alessandro A, Dueñas A, Gracián M, et al. Diarrheal diseases of infancy in Cali, Colombia: study design and summary report on isolated disease agents. Bull Pan Am Health Organ 1976;10:143-55. 
7. Jaramillo E, Estrada S, Ospina S. Etiología de la enfermedad diarreica aguda (EDA) de origen bacteriano, utilizando un protocolo estandarizado de laboratorio. Infectio 1999;3:95-9.

8. Muñoz NE, Agudelo $\mathrm{Cl}$, Ovalle MV, Realpe ME. Vigilancia en red de los serotipos y la susceptibilidad antimicrobiana de Salmonella sp., Shigella sp. y Vibrio cholerae 01, 1997-1999. Biomédica 2000;20:210-7.

9. Muñoz N, Agudelo Cl, Realpe ME, Ovalle MV. Vigilancia en red de la susceptibilidad antimicrobiana y de los serotipos de Salmonella spp., Shigella sp. y Vibrio cholerae: informe de 2000-2001. Inf Quinc Epidemiol Nac 2002;7:177-192.

10. Coll P. Interés de las técnicas de biología molecular en el estudio de las infecciones nososcomiales. Enferm Infecc Microbiol Clin 1994;12:369-71.

11. Chiou CS, Hsu WB, Wei HL, Chen JH. Molecular epidemiology of a Shigella flexneri outbreak in a mountainous township in Taiwan, Republic of China. J Clin Microbiol 2001;39:1048-56.

12. Ewing WH. Edward's and Ewing's identification of Enterobacteriaceae. 4th. ed. New York, NY: Elsevier Sciencie Publishing Co. Inc.; 1986.

13. Olive M, Bean P. Principles and applications of methods for DNA-based typing of microbial organisms. J Clin Microbiol 1999;37:1661-9.

14. National Committee for Clinical Laboratory Standards. Performance standards for antimicrobial testing. Eighth informational supplement. NCCLS document M100. Wayne, PA: NCCLS; 2001.

15. Swaminathan B, Barrett TJ, Hunter SB, Tauxe RV, CDC PulseNet task force. PulseNet: the molecular subtyping network for foodborne bacterial disease surveillance, United States. Emerg Infect Dis 2001;7:382-90.

16. Centers for Disease Control and Prevention. Standardized molecular subtyping of foodborne bacterial pathogens by pulsed field gel electrophoresis: a manual. Atlanta: National Center for Infectious Diseases; 1996 (updated 2000).

17. Tenover FC, Arbeit RD, Goering RV, Mickelsen PA, Murray BE, Persing DH, Swaminathan B. Interpreting chromosomal DNA restriction patterns produced by pulsed-field gel electrophoresis: criteria for bacterial isolate typing. J Clin Microbiol 1995;33: 2233-9.

18. Hall BG. Phylogenetic trees made easy. A how-to manual for molecular biologists. Sunderland, MA: Sinauer Associates, Inc.; 2001.

19. Cazentini MI, Nogueira S, Da Silva P, Capuano D, Errera MC, Fernandes S, et al. Etiology of acute diarrhea among children in Ribeirao Preto, SP, Brazil. Rev Inst Med Trop Sao Paulo 2001;43:1-8.

20. Cáceres DC, Acosta J. Evaluación de la situación de cólera en Colombia. Inf Quinc Epidemiol Nac 1998;4:18.

21. Sack RB, Rahman M, Yunus M, Khan EH. Antimicrobial resistance in organisms causing diarrheal disease. Clin Infect Dis 1997;24(Suppl. 1):102-5.

22. Tuttle J, Thomas KW, Tauxe RV. Shigellosis: treatment and prevention in native American communities. The Provider. Indian Health Service 1992;17:117-8.

23. DuPont HL, Levine MM, Hornick RB, Formal SB. Inoculum size in shigellosis and implications for expected mode of transmission. J Infect Dis 1989;159: 1126-8.

24. Trevejo RT, Abbott SL, Wolfe MI, Meshulam J, Yong D, Flores JR. An untypeable Shigella flexneri strain associated with an outbreak in California. J Clin Microbiol 1999;37:2352-3.

25. Morera MA, Espejo E, Coll P, Simón M, Uriz MS, Llovet T, et al. Epidemic outbreak of shigellosis following water intake. Enfer Infect Microbiol Clin 1995;13:160-5.

26. Yuk-Fong P, Lau YJ, Hu BS, Shyr JM, Shi ZY, Tsai WS et al. Analysis of clonal relationships among isolates of Shigella sonnei by different molecular typing methods. J Clin Microbiol 1995;33:1779-83.

27. Lima AAM, Sidrim JJC, Lima NL, Titlow W, Evans ME, Greenberg RN. Molecular epidemiology of multiple antibiotic-resistant Shigella flexneri in Fortaleza, Brazil. J Clin Microbiol 1997;35:1061-5.

28. Houang ETS, Chu YW, NG TK, Cheng FB. Study of the relatedness of isolates of Shigella flexneri and Shigella sonnei obtained in 1986 and 1987 and in 1994 and 1995 from Hong Kong. J Clin Microbiol 1998; 36:2404-7. 